\title{
Temperature-dependent lattice dynamics of antiferromagnetic and ferromagnetic phases of FeRh
}

\author{
M. P. Belov $\odot,{ }^{1}$ A. B. Syzdykova, ${ }^{1}$ and I. A. Abrikosov ${ }^{2}$ \\ ${ }^{1}$ Materials Modeling and Development Laboratory, National University of Science and Technology “MISIS,” Moscow, Russia \\ ${ }^{2}$ Department of Physics, Chemistry and Biology (IFM), Linköping University, SE-581 83 Linköping, Sweden
}

(Received 10 June 2019; revised manuscript received 22 January 2020; accepted 26 February 2020; published 16 April 2020)

\begin{abstract}
We have investigated lattice dynamics of antiferromagnetic and ferromagnetic phases of cubic (B2) FeRh at various temperatures from first principles using the temperature-dependent effective potential method. We have shown that already at low temperature the cubic structure of the antiferromagnetic phase becomes dynamically stable, which eliminates the contradiction between experimental observations and previous theoretical results showing its dynamical instability at temperature $T=0 \mathrm{~K}$. In addition, we have observed a significant difference in the temperature dependence of lattice vibrations of the ferromagnetic and antiferromagnetic phases. The phonon spectrum of the FM phase softens much stronger than that of the AFM phase, which provides additional contribution to the increase of vibrational entropy of the FM phase at high temperatures. The calculated difference between the vibrational entropies of the FM and AFM phases at a metamagnetic transition temperature $(350 \mathrm{~K})$ is $16 \mathrm{~J} / \mathrm{kg} / \mathrm{K}$. This value is comparable with the experimental value of the total entropy change. We therefore conclude that the lattice dynamics plays a decisive role in the metamagnetic phase transition in $\mathrm{FeRh}$ and its remarkable magnetocaloric properties.
\end{abstract}

DOI: 10.1103/PhysRevB.101.134303

\section{INTRODUCTION}

FeRh is a material that exhibits isostructural metamagnetic first-order phase transition from an antiferromagnetic (AFM) phase to a ferromagnetic (FM) phase above room temperature, at $T \sim 350 \mathrm{~K}[1,2]$. Experimentally it is well established that the FeRh alloy has the CsCl-type (B2) structure [3-6]. The low-temperature AFM state has type-II spin structure $[7,8]$. This functional material discovered more than seven decades ago [9-12] demonstrates remarkable properties, such as colossal magnetoresistance [13] and strong magnetostriction [14]. Consequently, the FeRh compound is of interest for multiple technological applications, including heat assisted magnetic recording (HAMR) $[15,16]$ and antiferromagnetic spintronics [17-19]. Moreover, an observation of a giant magnetocaloric effect [20] accompanied by a release or absorption of heat at a constant temperature makes FeRh into a highly promising material for magnetic refrigeration [21,22].

The magnetic phase transition is accompanied by a large total entropy change. The magnitude of the latter obtained in different measurements carried out for field-, pressure, and temperature-induced transitions ranges from 12 to $19 \mathrm{~J} / \mathrm{kg} / \mathrm{K}$ [1,5,23-29]. In the case of FeRh, it is customary to divide the full entropy change into electronic, lattice, and magnetic contributions. Early models suggested that the electronic entropy difference $\Delta S_{\text {el }}$ was the most important for the transition [30-32]. Recent work [13] also confirmed that entropy release at the transition is of electronic origin. However, this conclusion is in contradiction with electronic specific heat measurements, which revealed that the electronic contribution is small in comparison with the full entropy change $[23,33]$. Thus, more recent models mostly focused on the magnetic contribution $\Delta S_{\text {magn }}$ [34-36].
The lattice contribution was estimated many years ago, for instance, in Ref. [26]. The value of $\Delta S_{\text {latt }}$ was found to be much smaller than the full entropy change. However, in the experimental work of Cooke et al. [23] lattice contribution to total entropy change $(\Delta S=17 \pm 3 \mathrm{~J} / \mathrm{kg} / \mathrm{K})$ was deduced from the low-temperature specific heat measurements at temperatures below $20 \mathrm{~K}$. A large negative value of $\Delta S_{\text {latt }}=$ $-33 \pm 9 \mathrm{~J} / \mathrm{kg} / \mathrm{K}$ was obtained, implying that the lattice entropy change should impede the phase transition.

The lattice dynamics of both cubic AFM and FM phases was investigated in recent theoretical works in the framework of the density functional theory (DFT) $[24,37,38]$. Surprisingly, theoretical calculations at zero temperature predicted dynamical instability of the cubic AFM phase due to the presence of imaginary phonon frequencies at the Brillouin zone boundary. In addition, in Ref. [24] the lattice entropy change was calculated. In the case of the dynamically unstable cubic AFM phase the calculation of the entropy was performed on truncated phonon spectra neglecting its imaginary part. The authors concluded that lattice, electronic, and magnetic degrees of freedom contribute on roughly equal footing to the metamagnetic transition [24].

Thus, it is still actively debated which contribution to the total entropy change is driving the magnetic phase transition in FeRh. Therefore, the task of a consistent theoretical estimation of $\Delta S_{\text {latt }}$ contribution remains highly relevant. In this work we have addressed this challenging task. We have calculated phonon spectra at different temperatures employing the temperature-dependent effective potential (TDEP) method $[39,40]$ and demonstrated that the cubic AFM phase is, in fact, dynamically stable at nonzero, but still quite low, temperature. In addition, we have shown that the lattice entropy change has quite a large value, which is comparable with the experimental 
full entropy change. Our results demonstrate that $\Delta S_{\text {latt }}$, in fact, drives the metamagnetic transition in FeRh.

\section{COMPUTATIONAL DETAILS}

All calculations were performed in the framework of DFT using the Vienna $a b$ initio simulation package VASP [41-44]. The interactions between ions and electrons were described using the projector augmented-wave (PAW) method [44,45]. The exchange and correlation effects in the electron gas were treated within the generalized gradient approximation (GGA) employing the Perdew-Burke-Ernzerhof (PBE) [46] functional form. The energy cutoff of plane waves was chosen as $450 \mathrm{eV}$.

Four basis atoms in a fcc-like unit cell were chosen as an elementary cell. The integration over the Brillouin zone was performed using a gamma-centered mesh: a $9 \times 9 \times 9 k$ point grid for a $2 \times 2 \times 2$ (32 atom) supercell; a $6 \times 6 \times 6 k$ point grid for a $3 \times 3 \times 3$ (108 atom) supercell; a $5 \times 5 \times$ $5 k$-point grid for a $4 \times 4 \times 4$ (256 atom) supercell. All the calculations were carried out using the Methfessel-Paxton smearing (first order) method [47] with a broadening of $\sigma=0.1 \mathrm{eV}$.

Phonon calculations were performed in the harmonic approximation using the small displacement method $(0.01 \mathrm{~A})$ implemented in the PHONOPY code [48] at $T=0 \mathrm{~K}$. A recent comparison of various methods that allow one to take into account anharmonic effects of the lattice vibrations at finite temperature was given by Korotaev et al. [49]. In this work we employed the temperature-dependent effective potential (TDEP) method $[39,40]$. In this method, the main input is the displacements of atoms from their equilibrium positions and the forces generated due to these displacements. Such information is easily obtained, for instance from molecular dynamics simulations ( $a b$ initio or with model potentials).

In the framework of the second-order TDEP a true anharmonic potential energy landscape at a finite temperature is approximated by an effective harmonic potential. The difference between the true $a b$ inito forces $\mathbf{F}_{i}^{\mathrm{AI}}$ and in the forces obtained within the harmonic model is minimized:

$$
\min _{\tilde{\Phi}} \Delta \mathbf{F}=\min _{\tilde{\Phi}} \frac{1}{N_{c}} \sum_{i=1}^{N_{c}}\left|\mathbf{F}_{i}^{\mathrm{AI}}-\tilde{\Phi} \mathbf{U}_{i}^{\mathrm{AI}}\right| .
$$

Here $\mathbf{U}_{i}^{\mathrm{AI}}$ is a set of displacements vectors for all atoms for the $i$ th configuration from $N_{c}$ configurations used for the fitting of the effective second-order temperature-dependent force constants $\tilde{\Phi}_{i j}^{\alpha \beta}$. The latter are used for calculations of the phonon dispersion relations and the lattice entropy in the same way as in the harmonic approximation [50], but the calculated functions become temperature dependent through the temperature dependence of the TDEP force constants.

Additional TDEP calculations were performed at $T=0 \mathrm{~K}$ for comparison. In case of zero-temperature TDEP calculations the same input data as in PHONOPY calculations were used. At nonzero temperatures we used $N_{c}$ up to $\sim 200$ [40]. Configurations were obtained from molecular dynamics (MD) simulations at corresponding temperatures controlled with the Nosé thermostat [51] and carried out with a time step of $1 \mathrm{fs}$.
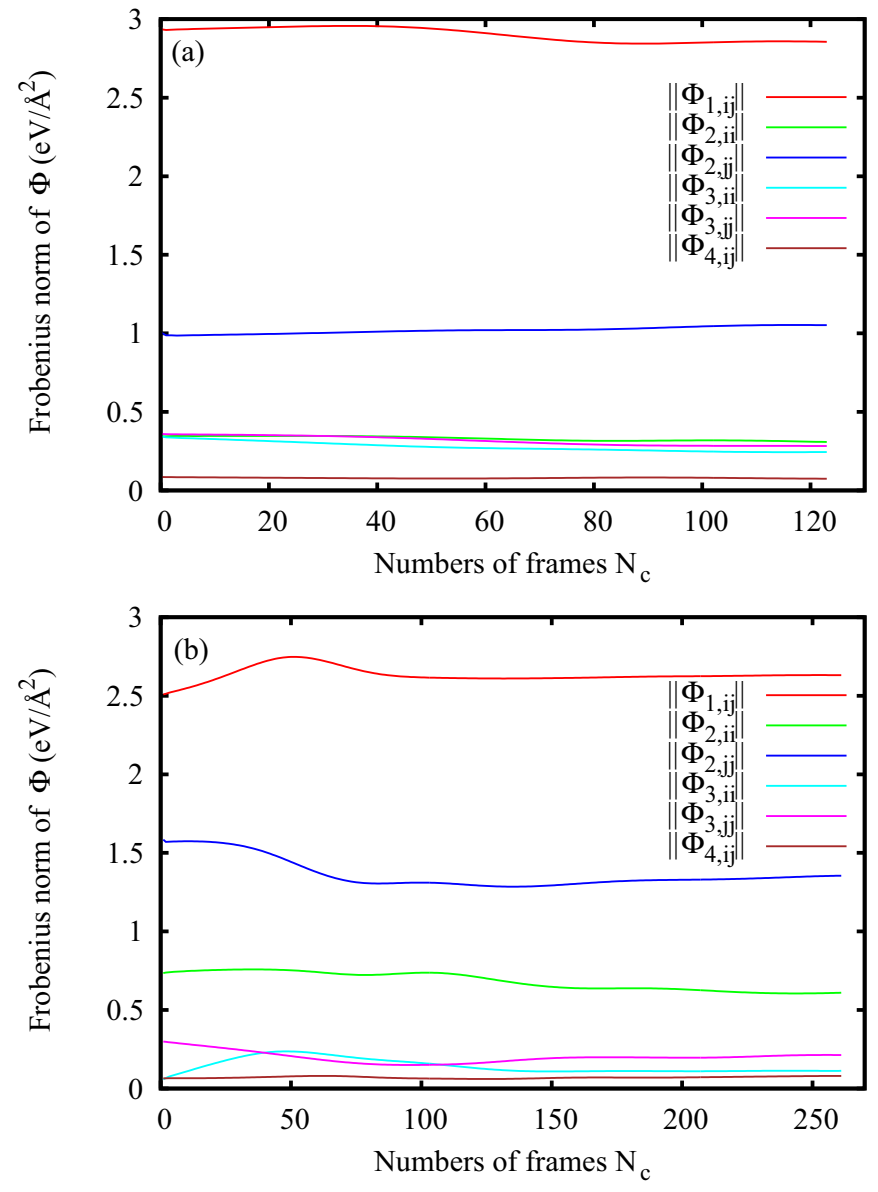

FIG. 1. Frobenius norm $\left\|\Phi_{n, i j}\right\|$ of force constants for each of the first four coordination shells (a) for the AFM phase at $100 \mathrm{~K}$ and (b) for the FM phase at $350 \mathrm{~K}$; here $n$ is the shell number and $i$ and $j$ define the sort of atom: Fe or Rh.

To demonstrate the convergence of our results, we have calculated the Frobenius norm of the force constants for each of the first four coordination shells for both the AFM and the FM phases. The results are shown in Fig. 1. One can see that for the AFM phase, the results converge quickly, while for the FM phase a larger number of configurations is required. This difference could be explained by the stronger anharmonic effects of lattice vibrations in the FM phase, as will be discussed below.

Note that according to the experimental data [52] the fractional change in lattice parameter of the AFM phase due to thermal expansion near $300 \mathrm{~K}$ is $\Delta a / a \sim 3 \times 10^{-3}$. Therefore, in our calculations we did not take into account the thermal expansion.

\section{RESULTS AND DISCUSSION}

Let us first discuss the calculated ground-state properties of the AFM and FM phases. Our results are summarized in Table I together with experimental data. A comparison shows good agreement between theory and experiment. Note that the properties summarized in Table I are well reproduced in the framework of the chosen computational setup. In addition, 
TABLE I. Comparison of equilibrium lattice parameter, local magnetic iron $m_{\mathrm{Fe}}$, and rhodium $m_{\mathrm{Rh}}$ moments with experimental data for two magnetic phases.

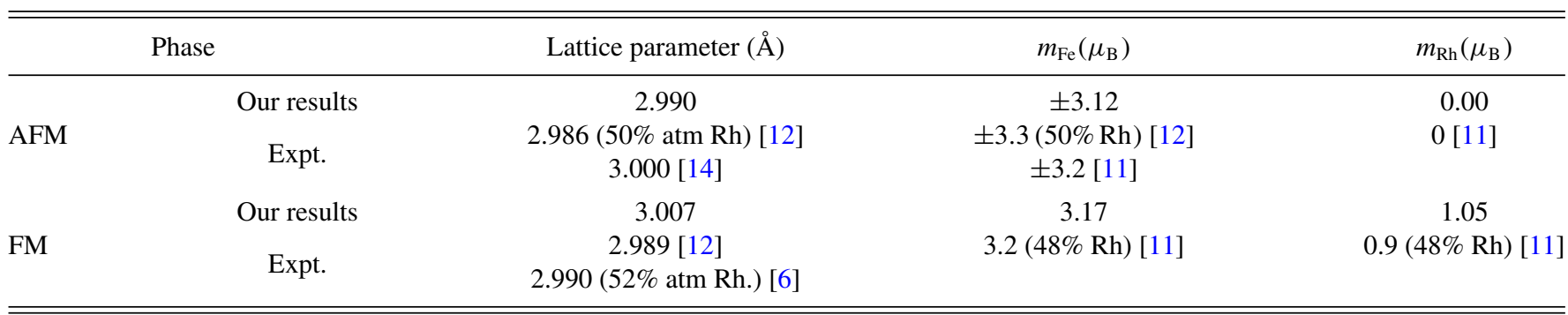

we observe good agreement with the parameters calculated in previous theoretical works $[24,37,38]$.

Let us next discuss the phonon dispersion relations calculated in the conventional harmonic approximation from DFT calculations at $T=0 \mathrm{~K}$. The results for the AFM and FM phases are presented in Figs. 2 and 3, respectively. Considering the convergence of the phonon dispersion relations with respect to the supercell size, it is clearly seen that for a $4 \times 4 \times$ 4 supercell, they converge well throughout the Brillouin zone for the both magnetic phases, except for narrow frequency regions near points $K$ and $U$ in the AFM phase. However, these regions are small. Moreover, it is seen that the strongest feature of the spectrum, the instability observed at the $X$ point in the AFM phase, is practically independent of the supercell size. We used a supercell with a size of $4 \times 4 \times 4$ in the subsequent calculations because an additional increase of a supercell size makes calculations too time consuming.

Note that the presence of imaginary frequencies near the $X$ point (Fig. 2) indicates the dynamic instability of the AFM phase and implies that the considered cubic structure should not exist at zero temperature. The dynamical instability was also observed in earlier theoretical calculations [24,37], and this may appear as a contradiction with the experimental data [3-6]. In Ref. [38] it was suggested that a modulated structure corresponding to this instability could be the ground state of the AFM phase and that the energy of the modulated structure

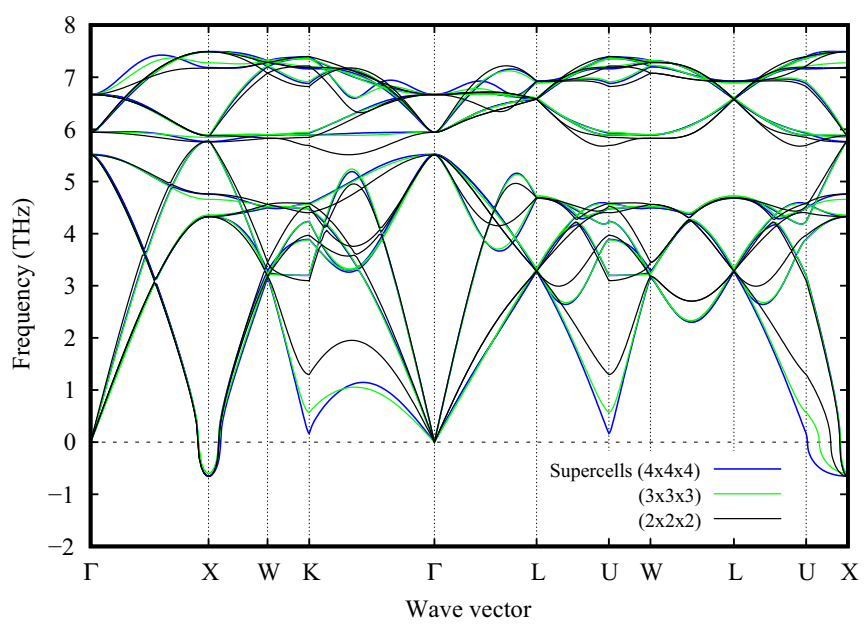

FIG. 2. Phonon dispersion relations calculated for the AFM phase of $B 2 \mathrm{FeRh}$ in the framework of the harmonic approximation at zero temperature using the supercells of different sizes. is less than the energy of the cubic structure by $0.125 \mathrm{meV}$. This energy difference is very small and therefore the entropic effects at low-temperature or even zero-point vibrations could stabilize the cubic structure. Thus, we have investigated the lattice dynamics of the cubic magnetic phases of FeRh at finite temperatures by the TDEP method $[39,40]$.

Phonon spectra of the AFM and FM phases of B2 FeRh as well as phonon density of states calculated at different temperatures are presented in Figs. 4 and 5, respectively. We note that using displacements and corresponding forces from small displacement calculations in the TDEP force constants fitting procedure $[39,40]$, the imaginary frequencies of the cubic antiferromagnetic phase at $T=0 \mathrm{~K}$ are also reproduced in the TDEP calculations (Fig. 4, red lines). With temperature increasing the frequencies of the corresponding branches become real, even at $T=100 \mathrm{~K}$ (Fig. 4, black lines), which is much lower than the magnetic transition temperature. A linear interpolation of the calculated frequencies at the $X$ point between $T=0 \mathrm{~K}$ and $100 \mathrm{~K}$ gives the estimation of the dynamical stabilization temperature of the cubic AFM phase at about $50 \mathrm{~K}$. In Ref. [53] the dynamical stabilization of the cubic structure was observed by means of the so-called large displacement method even at $T=0 \mathrm{~K}$. Here we provide a much more physical picture of the dynamical stabilization of the AFM phase of FeRh by anharmonic effects of the lattice

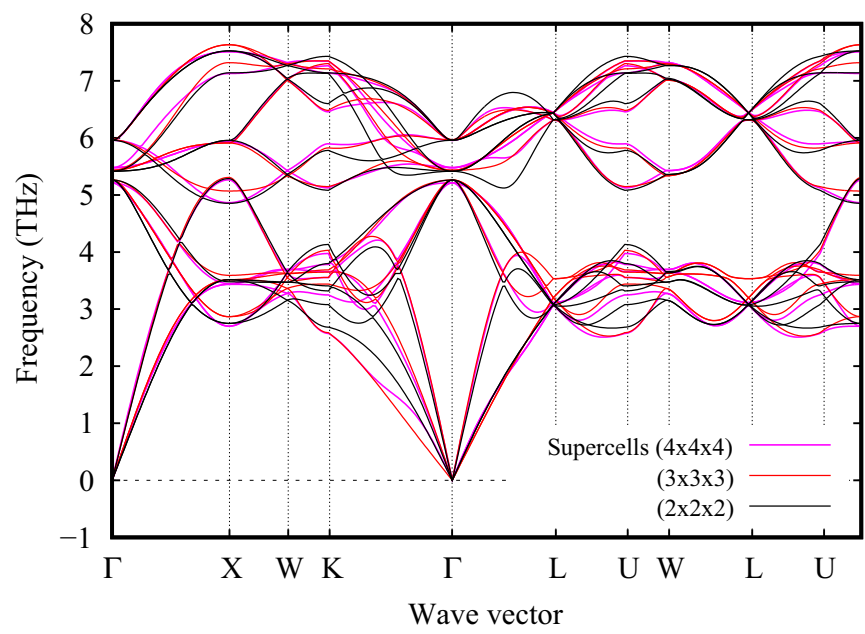

FIG. 3. Phonon dispersion relations calculated for the FM phase of $B 2 \mathrm{FeRh}$ in the framework of the harmonic approximation at zero temperature using the supercells of different sizes. 


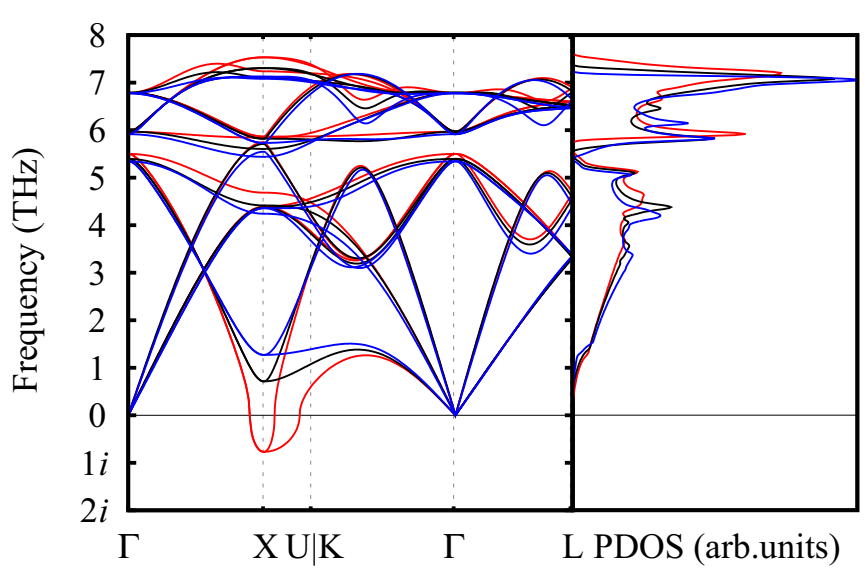

FIG. 4. Temperature dependence of the phonon dispersion relations and phonon density of states calculated for the AFM phase of $B 2 \mathrm{FeRh}$. Calculations have been carried out with the TDEP method for a $4 \times 4 \times 4$ supercell at $0 \mathrm{~K}$ (red lines), $100 \mathrm{~K}$ (black lines), and $350 \mathrm{~K}$ (blue lines). Small displacement method inputs for TDEP were used at $0 \mathrm{~K}$.

vibrations, and conclude that the temperature has a significant effect on the stabilization of the cubic phase. Therefore, the apparent contradiction between experiment and theory is resolved.

Note that while the anharmonicity of the AFM phase is strongly pronounced mainly for the $\mathrm{TA}_{1}$ branch of the $\Gamma-K \mid U-X$ direction of the Brillouin zone, the ferromagnetic phase is highly anharmonic in the entire Brillouin zone, as reflected by the strong temperature dependence of the entire phonon spectrum. Indeed, considerable softening of all the branches occurs with increasing temperature (Fig. 5).

We are now in the position to estimate the entropy associated with vibrational degrees of freedom for both magnetic phases of FeRh. Since we have calculated TDEP force constants at low $(0,100 \mathrm{~K})$ temperatures and at temperatures near magnetic phase transition $(350 \mathrm{~K})$, we can estimate

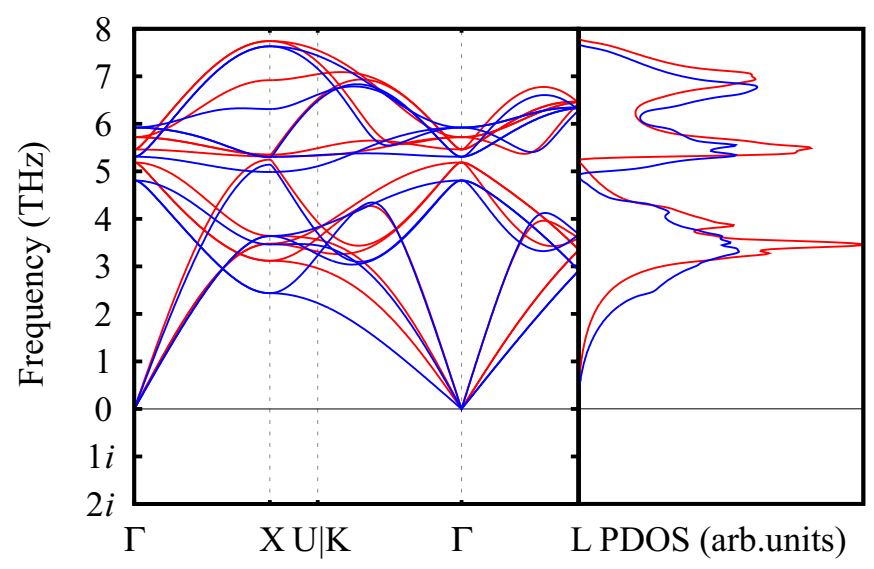

FIG. 5. Temperature dependence of the phonon dispersion relations and phonon density of states calculated for the FM phase of $B 2$ FeRh. Calculations have been carried out with the TDEP method for a $4 \times 4 \times 4$ supercell at $0 \mathrm{~K}$ (red lines) and $350 \mathrm{~K}$ (blue lines). Small displacement method inputs for TDEP were used at $0 \mathrm{~K}$.

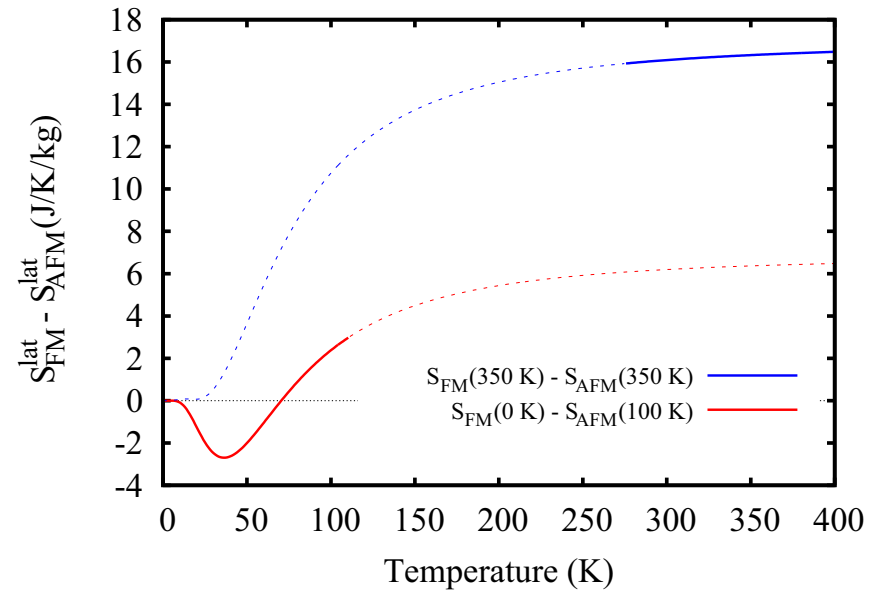

FIG. 6. Entropy difference of $B 2$ FeRh between AFM and FM phases obtained with low-temperature (red) and high-temperature (blue) TDEP force constants. Note that the AFM phase becomes dynamically unstable below $\sim 50 \mathrm{~K}$. Moreover, because of the strong anharmonic effects in $B 2 \mathrm{FeRh}$, the red curve gives a reliable estimation only at low temperatures, where we draw it as a solid line. On the contrary, the blue curve gives a reliable estimation of the entropy difference at high temperatures, which is also indicated by a solid line.

the difference in the vibrational entropies of phases at these temperatures quite accurately. Figure 6 shows the estimation of the temperature-dependent vibrational entropy difference. The red curve is obtained with the force constants calculated in the low-temperature limit, and the blue curve corresponds to the high-temperature force constants. At low temperatures (red curve) we obtained a negative entropy difference, which agrees with the experimental [23] and earlier theoretical data [53]. On the contrary, at temperatures close to the phase transition temperature (blue curve) the calculated difference of the vibrational entropies is positive and is about $16 \mathrm{~J} / \mathrm{kg} / \mathrm{K}$. This value is much larger than the values predicted in other theoretical works: $\sim 3.66 \mathrm{~J} / \mathrm{kg} / \mathrm{K}[53]$ and $\sim 5.85 \mathrm{~J} / \mathrm{kg} / \mathrm{K}$ [24]. This emphasizes the importance of taking into account the temperature dependence of atomic motions consistently. Most importantly, comparing our high-temperature result with experimental data on the total entropy change at the transition temperature, which vary from 12 to $19 \mathrm{~J} / \mathrm{kg} / \mathrm{K}$ [1,5,23-29], we conclude that the vibrational entropy is the dominant contribution to the total effect. Thus, the metamagnetic phase transition in FeRh is mainly due to the vibrational degrees of freedom.

\section{CONCLUSIONS}

We have shown that the dynamic instability of the cubic antiferromagnetic phase of $B 2 \mathrm{FeRh}$, which was found in a number of earlier theoretical works by means of DFT calculations at zero temperature, disappears at finite temperatures. Thereby we have removed the apparent contradiction between the experimental and theoretical data. Moreover, we have demonstrated that the lattice dynamics of both magnetic phases depends strongly on temperature. However, the implication of the anharmonicity is different in the AFM and 
FM FeRh. In the former phase the phonon spectrum softens slightly with temperature, except for the branch $\mathrm{TA}_{1}$ of the $\Gamma-K \mid U-X$ direction, which becomes stiffer. On the contrary, the entire spectrum of the ferromagnetic phase softens substantially with increasing temperature leading to the strong increase of the vibrational entropy of the FM phase near the metamagnetic transition temperature. Numerical estimation of the magnitude of the effect has allowed us to conclude that the contribution of vibrational degrees of freedom to the total change of entropy upon the metamagnetic transition should be dominant.

\section{ACKNOWLEDGMENTS}

Calculations of vibrational properties were supported by the Ministry of Science and High Education of the Russian Federation in the framework of the Increase Competitiveness Program of NUST "MISIS" (Grant No. K2-2019-001) implemented by a governmental decree dated 16 March 2013, No. 211. Theoretical analysis of the anharmonic effects was supported by the Russian Foundation for Basic Research (Grant No. 19-02-00871). Support from the Swedish Research Council (VR) through Project Grant No. 2019-05600 is gratefully acknowledged.
[1] M. Annaorazov, K. Asatryan, G. Myalikgulyev, S. Nikitin, A. Tishin, and A. Tyurin, Cryogenics 32, 867 (1992).

[2] L. J. Swartzendruber, Bull. Alloy Phase Diagrams 5, 560 (1984)

[3] C. Bordel, J. Juraszek, D. W. Cooke, C. Baldasseroni, S. Mankovsky, J. Minár, H. Ebert, E. E. Fullerton, and F. Hellman, Phys. Rev. Lett. 109, 117201 (2012).

[4] Q. J. Yap, J. J. Qiu, P. Luo, J. F. Ying, G. C. Han, D. E. Laughlin, J.-G. Zhu, T. Kanbe, and T. Shige, J. Appl. Phys. 116, 043902 (2014).

[5] A. Chirkova, K. Skokov, L. Schultz, N. Baranov, O. Gutfleisch, and T. Woodcock, Acta Mater. 106, 15 (2016).

[6] J. S. Kouvel and C. C. Hartelius, J. Appl. Phys. 33, 1343 (1962).

[7] V. L. Moruzzi and P. M. Marcus, Phys. Rev. B 46, 2864 (1992).

[8] R. S. Tebble and D. J. Craik, Magnetic Materials (Wiley Interscience, New York, 1969), p. 23.

[9] M. Fallot, Ann. Phys. 10, 291 (1938).

[10] M. Fallot and R. Hocart, Rev. Sci. 77, 498 (1939).

[11] G. Shirane, C. W. Chen, P. A. Flinn, and R. Nathans, Phys. Rev. 131, 183 (1963).

[12] G. Shirane, R. Nathans, and C. W. Chen, Phys. Rev. 134, A1547 (1964).

[13] M. A. de Vries, M. Loving, A. P. Mihai, L. H. Lewis, D. Heiman, and C. H. Marrows, New J. Phys. 15, 013008 (2013).

[14] M. R. Ibarra and P. A. Algarabel, Phys. Rev. B 50, 4196 (1994).

[15] D. Weller, G. Parker, O. Mosendz, E. Champion, B. Stipe, X. Wang, T. Klemmer, G. Ju, and A. Ajan, IEEE Trans. Magn. 50, 1 (2014).

[16] J. U. Thiele, S. Maat, and E. E. Fullerton, Appl. Phys. Lett. 82, 2859 (2003).

[17] T. Jungwirth, X. Marti, P. Wadley, and J. Wunderlich, Nat. Nanotechnol 11, 231 (2016).

[18] X. Marti, I. Fina, C. Frontera, J. Liu, P. Wadley, Q. He, R. J. Paull, J. D. Clarkson, J. Kudrnovský, I. Turek et al., Nat. Mater. 13, 367 (2014).

[19] R. O. Cherifi, V. Ivanovskaya, L. C. Phillips, A. Zobelli, I. C. Infante, E. Jacquet, V. Garcia, S. Fusil, P. R. Briddon, N. Guiblin et al., Nat. Mater. 13, 345 (2014).

[20] V. Franco, J. S. Blázquez, B. Ingale, and A. Conde, Annu. Rev. Mater. Res. 42, 305 (2012).

[21] B. Yu, M. Liu, P. W. Egolf, and A. Kitanovski, Int. J. Refrig. 33, 1029 (2010).

[22] M. Manekar and S. B. Roy, J. Phys. D: Appl. Phys. 41, 192004 (2008).
[23] D. W. Cooke, F. Hellman, C. Baldasseroni, C. Bordel, S. Moyerman, and E. E. Fullerton, Phys. Rev. Lett. 109, 255901 (2012).

[24] M. Wolloch, M. E. Gruner, W. Keune, P. Mohn, J. Redinger, F. Hofer, D. Suess, R. Podloucky, J. Landers, S. Salamon et al., Phys. Rev. B 94, 174435 (2016).

[25] E. Stern-Taulats, A. Planes, P. Lloveras, M. Barrio, J. L. Tamarit, S. Pramanick, S. Majumdar, C. Frontera, and L. Mañosa, Phys. Rev. B 89, 214105 (2014).

[26] J. S. Kouvel, J. Appl. Phys. 37, 1257 (1966).

[27] M. Richardson, D. Melville, and J. Ricodeau, Phys. Lett. A 46, 153 (1973).

[28] B. K. Ponomarev, JETP 36, 105 (1973).

[29] A. I. Zakharov, A. M. Kadomtseva, R. Z. Levitin, and E. G. Ponyatovskii, JETP 19, 1348 (1964).

[30] P. Tu, A. J. Heeger, J. S. Kouvel, and J. B. Comly, J. Appl. Phys. 40, 1368 (1969).

[31] C. Koenig, J. Phys. F 12, 1123 (1982).

[32] Y. Kobayashi, K. Muta, and K. Asai, J. Phys.: Condens. Matter 13, 3335 (2001).

[33] B. Fogarassy, T. Kemény, L. Pál, and J. Tóth, Phys. Rev. Lett. 29, 288 (1972).

[34] M. E. Gruner, E. Hoffmann, and P. Entel, Phys. Rev. B 67, 064415 (2003).

[35] R. Y. Gu and V. P. Antropov, Phys. Rev. B 72, 012403 (2005).

[36] L. M. Sandratskii and P. Mavropoulos, Phys. Rev. B 83, 174408 (2011).

[37] U. Aschauer, R. Braddell, S. A. Brechbühl, P. M. Derlet, and N. A. Spaldin, Phys. Rev. B 94, 014109 (2016).

[38] J. Kim, R. Ramesh, and N. Kioussis, Phys. Rev. B 94, 180407(R) (2016).

[39] O. Hellman, I. A. Abrikosov, and S. I. Simak, Phys. Rev. B 84, 180301(R) (2011).

[40] O. Hellman, P. Steneteg, I. A. Abrikosov, and S. I. Simak, Phys. Rev. B 87, 104111 (2013).

[41] G. Kresse and J. Hafner, Phys. Rev. B 47, 558 (1993).

[42] G. Kresse and J. Hafner, Phys. Rev. B 49, 14251 (1994).

[43] G. Kresse and J. Furthmuller, Comput. Mater. Sci. 6, 15 (1996).

[44] G. Kresse and D. Joubert, Phys. Rev. B 59, 1758 (1999).

[45] P. E. Blochl, Phys. Rev. B 50, 17953 (1994).

[46] J. P. Perdew, K. Burke, and M. Ernzerhof, Phys. Rev. Lett. 77, 3865 (1996).

[47] M. Methfessel and A. T. Paxton, Phys. Rev. B 40, 3616 (1989).

[48] A. Togo, F. Oba, and I. Tanaka, Phys. Rev. B 78, 134106 (2008). 
[49] P. Korotaev, M. Belov, and A. Yanilkin, Comput. Mater. Sci. 150, 47 (2018).

[50] G. Grimwall, Thermophysical Properties of Materials (Elsevier Science B.V., Amsterdam, 1999).

[51] S. Nosé, Prog. Theor. Phys. Suppl. 103, 1 (1991).
[52] J. B. McKinnon, D. Melville, and E. W. Lee, J. Phys. C: Solid State Phys. 3, S46 (1970).

[53] N. A. Zarkevich and D. D. Johnson, J. Alloys Compd. 802, 712 (2019). 